\title{
Exploration of BIM Video Diversification Teaching Based on the Theory of MOOC Research
}

\author{
Jingjing Chen* \\ School of Management and E-business \\ Zhejiang Gongshang University \\ Hangzhou, China
}

\author{
Juan Wu \\ School of Management and E-business \\ Zhejiang Gongshang University \\ Hangzhou, China
}

\begin{abstract}
The diversification of teaching videos will improve the enthusiasm and efficiency of learning. The main purpose of the paper is to get the training of BIM short video and considering the learner's psychological and environmental factors. Therefore, this paper firstly combined the willingness research data of multi-course platform, the student data provided by Thsware Company and the profit model research data before short video studio, secondly used the model analysis method to obtain comprehensive results, thirdly investigated the learning status and problems of some college students, then tried to find out the key factors affecting their learning based on empirical data, finally explored the influence mechanism of learning willingness in order to promote and improve their learning efficiency. The innovation of this paper lies in the comprehensive analysis of the internal and external factors affecting students learning on MOOC and the factors related to the surrounding environment, then put forward that BIM short video teaching experience training and platform technology upgrading, to meet the value needs of students.
\end{abstract}

Keywords-MOOC; learning retention; BIM

\section{INTRODUCTION}

The training service provided by BIM flash studio is different from the existing network training form, that is, online video education based on short video and student problem orientation. At present, the training market of BIM online courses is mostly based on the medium and long-term videos imparted by conventional knowledge (medium and long videos generally 5-20 minutes, long videos more than 20 minutes) or the form of live network broadcasting. There are no problemoriented training courses for short or ultra-short courses yet. Flash studios take the lead in adopting this mode, and with the help of partners. Strong comprehensive background, in technology, channels, social impact to achieve a clear firstmover advantage.

Based on the characteristics and advantages of online education, network classroom has been recognized by more and more people in recent years [1]. The "one-size-fits-all" teaching method can't accurately satisfy different personalities, nor can it maximize the teaching efficiency. We should make full use of the "Internet + BIM" technology to reform and innovate, and build core competitiveness. Raise the overall level of educational productivity. At present, there are more and more new online schools and related websites, such as the Three Bars Network, 91 Foreign Teachers Network and so on. Obviously, this represents that the network school has

Sponsor: Education and Science Planning Project of the Education Department of Zhejiang Province gradually entered public life and become a mainstream trend of learning. As a result, many people began to accept the form of online classroom teaching, especially white-collar workers and college students. In 2018, China's online education market share exceeded 300 billion yuan, and the scale of online education users was enlarged.

With the continuous development of building information model (BIM) technology and online education, they are also slowly combining. The scale of BIM competition in Colleges and universities in China is growing, and the demand for BIIM network training is growing [2]. At present, the training form provided by Thsware Company is mainly online medium-long video tutorials and studios. The short lessons have strengthened Thsware's overall strength.

\section{RESEARCH REVIEW}

\section{A. Teaching methods}

Users can get points by uploading short videos of difficult problems encountered in the process of using BIM software and self-made solutions to difficult problems. In addition, users can also get a considerable number of points through activities such as APP check-in, which can be converted into video viewing rights. In addition, members of BIM flash studio can watch all videos free of charge, otherwise, each video will be charged a certain viewing fee. Co-construction and sharing of resources: users can access, upload, download, store and use the resources in the library anytime and anywhere through the network or Flash course [3].

In addition to the functions of viewing, uploading and downloading short videos, BIM flash course APP also has the function of community discussion, which provides convenient and fast discussion channels and places for users to solve BIM difficult problems. This will deepen the stickiness between BIM flash course studio and user groups, and make more accurate viewing through users' use of information. Frequently recommended. In addition, APP will also provide users with online answering services anytime, anywhere.

Communication level. Online communication: providing BBS educational forum service, online answering questions, resource reviews and micro-blog speeches, etc. to realize online or offline-online communication and counseling for online teaching. BIM flash studio will arrange people to communicate with users online, collect valuable suggestions from users through BBS forum and micro-blog, constantly 
improve our web services and APP functions, and strive to provide better learning and communication experience for users [4].

\section{B. Technology control}

At present, the studio has obtained the right to use the BIM series patented technology in Swell, including: architectural design software, equipment design software, energy-saving design software, sunshine analysis software, lighting analysis software, HVAC load software, UniBIM platform, UniBIM for Review software, three-dimensional calculation for CAD, installation for CAD, three-dimensional calculation for CAD. The right to use calculation for Revit, installation for Revit, list valuation software, project management software, threedimensional site layout software, BIM 5D software, etc. At the same time, it has obtained the authorization of the shortbroadcast course [5].

The construction of the studio's resource base is mainly aimed at the difficult and miscellaneous points, which can't be obtained by the general way of information collection. It mainly comes from the information feedback collected by Swell in a large number of routine training, including the national competition undertaken by Swell, Swell College training, Swell enterprise training, Swell BIM certification training, etc.

\section{MEthODOLOGY}

This passage mainly explains the importance and rationality of short video teaching.

\section{A. Research approach}

In the field of acceptance research, the most influential technology integration model and planned behavior theory should be TAM, which contains two basic elements: perceived usefulness and perceived ease of use, but its own shortcomings lead to limited explanatory power. Venketech, Morris and others put forward the so-called Unified Theory of Acceptance and Use of Technology (UTAUT) on the basis of summing up the previous research on TA M. The empirical results show that UTAUT is better than any previous technology. The models are more effective, explaining $70 \%$ variation in usage behavior [6]. The technology integration model analyses the behavior of users intention from four core dimensions: performance expectation, effort expectation, social impact and convenience. Performance expectation is the extension and development of perceived usefulness [7]. It emphasizes the expectation of expected results and whether learning can bring about direct learning effects. Theory of Planned Behavior (TPB) is proposed by Ajzen on the basis of rational behavior theory. The theory of planned behavior can help us understand how people change their behavior patterns. TPB believes that human behavior is the result of well-thought-out planning. The three elements of planned behavior theory are attitude, subjective norm and perceived behavior control [8]. Among them, attitude describes a subjective tendency; subjective norms refer to the social pressure individuals feel, similar to social impact; perceived behavior control refers to the individual's self-control ability. The three factors together affect the behavioral intention of the actor.

The two primary goals of this study are to develop and extend existing business value theory to provide a market basis for short video production. At the same time, the theory also solves the problem of market value to realize the diversification of short videos and provide better teaching templates for students. Through the existing studio business value foundation to lay the foundation for short video education, thus proving the effectiveness of short video teaching diversification. In the first stage, substantive research. To study the participant's problems in learning exploration and the industrial problems covering different lines of businesses. Phase II, investigating the video teaching mode of Swell Company examines the basic dimensions of teaching that have been constructed. And then learn about the national participants learning situation and level.

\section{B. Data collection and sampling frame}

The existing BIM online courses take a long time and are divided according to the content section, so the comprehensive cross-cutting is poor. When students need to specialize in certain knowledge difficulties (including comprehensive difficulties or cross-cutting difficulties), the current online courses will not provide services, which greatly reduces the learning effect of the students.

Online courses generally charge higher fees. Some students only want to learn some knowledge points and need to buy long videos in full. Therefore, short videos are urgently needed to improve learning efficiency, reduce learning costs and solve the urgent problems for students.

The BIM training industry started late, with mixed industry, the training enterprise's teaching force may not be strong, and the price positioning varies. The most critical thing is that the BIM training institutions which have not been widely recognized for their reputation and integrity have not yet been produced.

Through cooperation with Swell Company, the studio collects debris problems in related technical training of various BIM competitions sponsored by Swell Company, and systematically collates and classifies them, so as to establish the corresponding short video library, and in the latter operation, continuously integrate various issues uploaded by users, gradually. Fill and improve the short video library. Compared with the general online education network classroom, the short video library built by the studio has more pertinence in dealing with problems, and different from the long-term online courses in the traditional network classroom, BIM flash studio solves difficult problems by a short video, users can use fragmented time anywhere and anytime [9].

\section{Measures}

The studio provides users with precise solutions in the form of short video libraries. Fees are paid for by membership fees and video resources. The integral system serves to build and share resources. Through the joint efforts of users and studios, short video libraries will be continuously improved. In addition, 
APP will be used to increase the number of short video libraries. Add user stickiness.

Overall, compared with the traditional online education enterprises, the studio has a fixed number and scale of customers every year. Based on the problem-oriented, it focuses on the production of BIM short-video teaching resource bank, and locates itself on the professional targeted network learning platform of BIM technical service. Through cooperation with Swell Company, the studio collects debris problems in related technical training of various BIM competitions sponsored by Swell Company, and systematically collates and classifies them, so as to establish the corresponding short video library, and in the latter operation, continuously integrate various issues uploaded by users, gradually. Fill and improve the short video library.

Compared with the general online education network classroom, the short video library built by the studio has more pertinence in dealing with problems, and different from the long-term online courses in the traditional online classroom, the studio solves difficult problems by a short video, users can learn photos anytime, anywhere through fragmented time. The content of the customs.

\section{CASE STUDY}

\section{A. Distinguishing the fundamental needs of students}

Situational attention to metadata and the operation of micro-particles can't be separated from the support of learning theory at the physiological level. The information of students is mainly based on the following analysis of online video learning resources in the process of learning behavior.

1) The first point is the cognitive load theory. Cognitive load refers to the matching between the amount of information presented and the size of working memory. The core hypothesis of cognitive load theory is working memory, which can only deal with limited information. If the learning task is too complex, it may be overloaded. So the trend of short video will make students improve their acceptance of learning. External cognitive load, also known as invalid cognitive load, is mainly caused by improper design of learning materials or inappropriate presentation in instructional design. Therefore, teachers should consider more rigorously reasonable instructional design when making a short video.

2) Second, the sense of social existence. It refers to the degree to which a person is regarded as a "real person" in the process of utilizing the media. That is to say, the media gives people the perception of social emotions. The sense of social existence is also called the auxiliary sense of remote presence or the social sense of remote presence. Picciano defines the sense of social existence as the student body based on the background of the network curriculum. Experience the feeling in the classroom, as well as the ability to interact with other students and teachers, so teachers should set the content of the short video as feedback.

\section{B. Establishment of the data collection system}

According to the information status and business needs of the company brought by trainees and other customers, the studio is integrated with the company's joint application system. There are generally two ways to realize the integration of heterogeneous databases in various systems. The first is to transplant the original data into the new data management system. In order to integrate different types of data, it is necessary to transform the data type. Middleware integration technology is usually used. Middleware, as a solution to the integration of exotic heterogeneous data sources, must choose a global data mode for middleware. The global model of heterogeneous data integration should satisfy the following requirements:(1) It can describe various data formats, whether structured or unstructured. (2) It is easy to exchange data, and the integrated data can be easily exchanged in various formats.

The key technology of middleware integration of heterogeneous databases is the synchronization of the heterogeneous databases. The synchronization of the database is for different administrators to share and analyze the data of trainees in real-time and integrate the information of trainees in an all-round way. In order to synchronize heterogeneous databases, there must be data coding standards and data coding must be unique, which is the basic requirement of data acquisition in the inheritance process of trainee information systems.

In the process of enterprise information construction, PDM system is the source data of all application systems. PDM system contains data information on all products. PDM system such as CAPP, ERP, MES provides EBOM tables. Other systems will be applied on the basis of BOM tables of PDM system. However, in the process of information construction, enterprises often implement ERP system in advance, and then implement PDM system. Select and adjust, and then unify the identification to achieve information synchronization.

\section{Establishment of feedback platform for students}

Different from traditional long video teaching methods, short video production is not simply a short copy of the long video.

To the Internet, but combined with the characteristics of network learning users elaborate, short video design and classroom

Participation is similar to a classroom. Specifically speaking, the open mode and transmission of short video courses

Offline courses are similar to regular open courses and appear as a new form of consumption, but combined with the characteristics of fragmented reading in the network age, the time of a single course usually varies from a few minutes to ten minutes. During the course, there will be corresponding small questions. Participants must answer the questions correctly in order to continue the course. If there are any questions in the course of learning, they can exchange and discuss it in the course forum. 


\section{SUMMARY}

\section{A. Consolidating the learning style from the short video teaching}

First, Participants can choose courses according to their own conditions and translate their desire for learning into practical action. College students choose curriculum resources independently, adjust learning content, learning strategies and learning processes actively according to the current state. This whole process is the outstanding manifestation of learning autonomy. Short video teaching mode includes participation, feedback, homework, discussion and other complete teaching modes, which can meet the needs of users for knowledge acquisition, and even help them quickly acquire urgent knowledge to meet immediate needs. While acquiring knowledge, we should cultivate their self-control and selfmanagement abilities.

Second, improving learning autonomy. Targeted short videos provide college students with the conditions for lifelong continuous learning. If the participants can confidently and fully invest in curriculum learning, they can constantly improve their learning methods and enhance their learning ability in the learning process; through the learning results obtained, they can make internal attribution and affirm themselves. Selflearning Ability to Improve Learning Autonomy

Third, Compared with the relatively complete time of study and activity, fragmentation time is fragmentary, but the sum of time accumulated over time will be a very large number. Short video platform can effectively utilize fragmented time, and greatly improve the time utilization and learning efficiency. Compared with long-term video teaching, short-term video teaching is spread through the Internet, and each course lasts only a few minutes. Under this premise, college students can learn knowledge and complete self-improvement as long as they have free time. The freedom of learning time and space in short video courses relaxes the restrictions on learning conditions.

Through the three points, we can know that as many new things, the impact of short videos on the learning style of trainees is twofold, with both opportunities and challenges. While bringing more learning resources and convenience, it also brings corresponding problems. Therefore, we should take an optimistic attitude to make good use of this short video teaching platform, and at the same time, we should take a cautious attitude to avoid the emergence of negative effects. For College students, any form of new media learning is a complement to traditional teaching. We should correctly recognize and select short video content according to our own needs, so as to improve the efficiency and effect of learning.

\section{B. Contributions}

Strict implementation of teacher's teaching norms. We should timely revise, supplement and improve the teaching rules and regulations on teaching construction and reform, teaching quality monitoring and other aspects, and form a training management system that conforms to the training of modern educational ideas and has the school's own characteristics, so as to promote the comprehensive quality and overall level of future BIM engineers.

Perfecting the whole teaching system. We will further improve the teaching objective system, the teaching quality standard system, the primary school information collection system, the teaching quality assessment system, the teaching information feedback system and the teaching quality monitoring and assurance system, so as to realize the full participation and supervision of the teaching quality management, so as to ensure the steady improvement of the teaching quality. The teaching objective system is composed of talent training objectives and curriculum system, which plays a normative and guiding role in the whole teaching process. According to the feedback of education information, the company judges the quality of short video teaching, affirms the positive part of the work, finds out the reasons for the weak links and rectifies them in time. At the same time, according to the social needs and the actual situation of company training, change the way of short video production, adjust the setting of short video shooting.

\section{REFERENCES}

[1] Wei Yingling, He Kunda. Current situation and development of MOOCs in European Union and Its Enlightenment to MOOCs in China [J]. Journal of Distance Education, 2015, pp.30-37, In Chinese.

[2] Qudi. Cold Reflections on MOOC Hot - International Reflections on the Six Major Issues of MOOCs Teaching [J]. Distance Education Research, 2014, pp.39-47, In Chinese.

[3] Zhang Qianfan, Wang Chengyu, Zhang Yajun. Empirical study on factors influencing MOOC learning intention of college students [J]. Higher education exploration,2015, pp.66-70, In Chinese.

[4] Luo Shuping, Xu Guifang. Application of BIM technology in architectural design and construction [J].Value Engineering,2018, pp.176-177, In Chinses.

[5] Le Yun, Zheng Xian, Li Yongkui, Lu Yujie, Bai Ju. Value stream and driving path of BIM technology application based on SVN [J]. Journal of Management Engineering, 2018, pp.71-78, In Chinses.

[6] Wu Shenghai, Liu Shannan, Liu Yongjia, Xu Zheng. Application and Analysis of BIM Technology Based on Dynamo Visual Programming Modeling [J]. Industrial Architecture, 2018, pp.35-38, In Chinses.

[7] Venkatech V, Morris M G, Davis G B, et al. User acceptance of information technology; toward a unified view[J]. MIS Quarterly, 2003, pp.425-478.

[8] Ajzen I. Perceived behavioral control, self-efficacy, locus of control, and the theory of planned behavior [J]. Journal of Applied Social Psychology,2002, pp.165-183.

[9] Bandura A. Self-efficacy: The Exercise of Control [M], New York: Freeman,1997, pp.122-134. 